\title{
Grundstein für die Parlamentsforschung im EU-Mehrebenensystem nach Lissabon
}

Abels, Gabriele und Annegret Eppler (Hrsg.): Auf dem Weg zum Mehrebenenparlamentarismus? Funktion von Parlamenten im politischen System der EU (Schriftenreihe des Arbeitskreises Europäische Integration e.V., Band 75), Nomos Verlagsgesellschaft, Baden-Baden 2011, 332 Seiten, $€ 69$,-

Dittgen, Friederike: Die europapolitische Rolle der nationalen Parlamente nach Lissabon (Würzburger Universitätsschriften zu Geschichte und Politik, Band 15), Nomos Verlagsgesellschaft, Baden-Baden 2011, 105 Seiten, $€ 22,-$.

Die demokratische Legitimation der Europäischen Union ist seit Jahrzehnten Gegenstand wissenschaftlicher Debatten. Durch den Vertrag von Lissabon und das Lissabon-Urteil des Bundesverfassungsgerichts hat diese Thematik weiter an Bedeutung gewonnen, wobei in Bezug auf Parlamentarisierungsstrategien mittlerweile Konsens herrscht, dass das europäische Mehrebenensystem seine Legitimität über verschiedene Kanäle demokratischer Repräsentation bezieht. Die beiden hier zu besprechenden Bücher beleuchten somit einen hochaktuellen Prozess. Während der Fokus bei Friederike Dittgen dabei auf den nationalen Parlamenten liegt, ist das Ziel von Gabriele Abels und Annegret Eppler, alle Parlamente im EU-Mehrebenensystem abzudecken.

Um die Frage zu beantworten, ,inwieweit der Lissabonner Vertrag tatsächlich eine verbesserte Grundlage für die europapolitische Beteiligung der nationalen Parlamente schafft" (S. 13), thematisiert Dittgen zunächst die Notwendigkeit dualer parlamentarischer Beteiligung in EU-Angelegenheiten. Anschließend skizziert sie die allgemeine historische Entwicklung der Mitwirkungsrechte nationaler Parlamente, ehe der Verfassungskonvent aufgearbeitet wird. Die Detailliertheit dieser Ausführungen erscheint dabei nicht immer zielführend und mitunter redundant, da im vierten Kapitel des Buches der Vertragstext ebenfalls genau unter die Lupe genommen wird. In diesem sowohl in Bezug auf Umfang als auch Inhalt Hauptkapitel des Buches werden die Neuerungen, die sich durch den Vertrag von Lissabon für die Beteiligung nationaler Parlamente ergeben, sehr genau und anschaulich verdeutlicht sowie kritisch analysiert. Der Schwerpunkt liegt dabei eindeutig auf dem neu geschaffenen Frühwarnmechanismus, dessen Schwierigkeiten in der Umsetzung vor allem durch die Schilderung eines vorab unter der Leitung der COSAC durchgeführten Subsidiaritätstestlaufs deutlich werden. Das anschließende Kapitel, das einen Eindruck „aus der Praxis innerstaatlicher Beteiligungsverfahren“ (S. 14) am Beispiel von Bundestag und Bundesrat geben soll, verharrt vorwiegend in einer Analyse der rechtlichen Grundlagen der europapolitischen Arbeit. Hier wäre eine umfangreichere Fallstudie, in der auch erste Ergebnisse zur tatsächlichen Wahrnehmung der Rechte durch Bundestag und Bundesrat präsentiert werden, hilfreich gewesen. Das nächste Kapitel, das sich dem Lissabon-Urteil des Bundesverfassungsgerichts widmet, hätte man aufgrund der rechtlichen Implikationen bereits vor den kurzen Ausführungen zur Mitwirkung von Bundestag und Bundesrat in EU-Angelegenheiten erwartet. Wenig überraschend bilanziert Dittgen schließlich, dass „der Lissabonner Vertrag ohne Zweifel einen Gewinn für die nationalen Parlamente dar[stellt]“ (S. 91), deren Umgang mit den rechtlichen Regelungen jedoch „eine langfristige Herausforderung“ (S. 92) bleibt.

Insgesamt vermittelt das Buch von Dittgen aufgrund einer detaillierten Untersuchung der europäischen und nationalen Vertragstexte einen umfassenden und verständlichen Überblick 
über die rechtlichen Neuerungen, die sich aus dem Vertrag von Lissabon für die nationalen Parlamente, vor allem in Deutschland, ergeben. Aufgrund der Tatsache, dass sich darüber hinausgehende Ausführungen vor allem auf Sekundärliteratur stützen, wird das Analysepotenzial im Hinblick auf die parlamentarische Mitwirkung in der „täglichen“ europapolitischen Arbeit bei weitem nicht ausgeschöpft.

Ziel des von Abels und Eppler herausgegebenen Tagungsbandes ist es, „die Funktionen von Parlamenten im EU-Mehrebenensystem sowohl empirisch als auch theoretisch genauer zu analysieren, eine Zwischenbilanz der Reformprozesse der letzten Jahre im Kontext des Lissabon-Vertrags zu ziehen sowie schließlich Mehrebenenparlamentarismus als theoriefähiges Konzept zu reflektieren“ (S. 18 f.). Die Herausgeberinnen führen dazu zunächst in wichtige theoretische Konzepte und wissenschaftliche Debatten ein und schaffen damit den Rahmen für die weiteren Beiträge - eine nicht ganz einfache Aufgabe, da die 17 verschiedenen Aufsätze von Wissenschaftlern verschiedener Disziplinen sowie Praktikern stammen und somit sehr vielfältige Aspekte beleuchten. Schließlich nehmen Abels und Eppler recht detailliert eine Aufteilung des Tagungsbandes in sieben Abschnitte vor.

Die ersten beiden Beiträge beschäftigen sich zunächst grundsätzlich mit konzeptionellen und methodologischen Perspektiven des Mehrebenenparlamentarismus, worunter sehr allgemein Strategien der Parlamentarisierung auf mehreren Ebenen gefasst werden. Andreas Maurer, der als Urheber dieses im Mittelpunkt der Tagung stehenden Terminus gelten darf, beschäftigt sich daher in seinem Beitrag mit der parlamentarisch-demokratischen Struktur in der EU und den Veränderungen durch den Vertrag von Lissabon. Katrin Auel arbeitet anschließend methodologische Herausforderungen für die Parlamentarismusforschung heraus und gibt einen umfassenden Überblick über bestehende Konzepte; die Frage, ob Mehrebenenparlamentarismus mehr als nur eine Metapher ist und somit beispielsweise über das von Ben Crum und John Fossum (2009) entwickelte Konzept des Multilevel Parliamentary Field hinaus Relevanz entwickelt, bleibt jedoch offen.

Die Beiträge im folgenden Abschnitt befassen sich mit der europäischen Ebene. Stefan Götze und Berthold Rittberger analysieren die kontinuierliche Ausweitung der Befugnisse des Europäischen Parlaments unter Rückgriff auf eine soziologisch institutionalistische Erklärung. Die Erweiterung der Kompetenzen wird demnach von den meisten Mitgliedsregierungen mittlerweile als „angemessene“, weil legitime Antwort auf die funktionale Ausweitung der Kompetenzen der EU betrachtet. Jürgen Mittags Beitrag zu den europäischen Parteien untersucht die Implikationen, die sich aufgrund der Revision der finanziellen Bestimmungen sowie der Etablierung politischer Stiftungen auf europäischer Ebene ergeben. Hierbei wäre neben einer Analyse der sehr spezifischen formalen Änderungen die genauere Betrachtung informeller Prozesse und Beziehungen sicherlich aufschlussreich gewesen.

Der dritte Abschnitt greift die Problematik der Exekutivdominanz auf: Für Timm Beichelt ist die Tatsache, dass Regierungen in der EU als quasi-parlamentarische Akteure agieren und somit als europäische Primärgesetzgeber gelten können, je nachdem ob ein intergouvernementales oder supranationales Leitbild zugrunde gelegt wird, aus legitimationstheoretischer Sicht unterschiedlich zu bewerten. Interessant, wenn auch sicherlich kontrovers, ist seine Forderung, den Rat als Gesetzgeber ebenfalls im Konzept des Mehrebenenparlamentarismus zu berücksichtigen. Martin Große Hüttmann und Michèle Knodt zeigen auf, dass die im Vertrag von Lissabon verankerte Strategie des Mehrebenenparlamentarismus im Fall der Euro-Krisenpolitik aufgrund der Dominanz der Regierungen keine legitimatorische Kraft entfalten konnte. Diesen Teil abschließend gibt Carmen Preising aus ihrer Sicht als stellver- 
tretende Referatsleiterin im Generalsekretariat der Europäischen Kommission eine äußerst interessante, an manchen Stellen jedoch allzu positive Zwischenbilanz der praktischen Umsetzung der Subsidiaritätskontrolle durch nationale Parlamente.

Im folgenden Abschnitt werden die Auswirkungen des Vertrags von Lissabon für die deutschen Parlamente herausgearbeitet: Matthias Niedobitek macht in seinem Beitrag zur Integrationsverantwortung von Bundestag und Bundesrat deutlich, warum das LissabonUrteil als politisches Urteil gelten kann und welche rechtlichen Einwände sich hieraus ergeben. Sven Vollrath sowie Ute Müller schildern die praktische Umsetzung der neuen Rechte durch Bundestag und Bundesrat, wobei Vollrath dabei anhand neuer, durch das Europareferat des Bundestags erhobener Daten einen äußerst interessanten Überblick über die europapolitischen Aktivitäten des Bundestags gibt.

Zwei Beiträge widmen sich dem Wandel parlamentarischer Rollen mit Fallstudien von jeweils drei Mitgliedstaaten (Deutschland, Frankreich und Polen bei Carina Sprungk sowie Deutschland, Schweden und Ungarn bei Sabine Kropp, Jonas Buche und Aron Buzogány). Sprungk analysiert die Herausbildung von drei neuen EU-spezifischen parlamentarischen Rollen; Abgeordnete sind demnach zusätzlich „Netzwerker“, „Integrationswächter“ und üben einen „EU-spezifischen Kontrollmodus“ aus. Sie ist sich dabei der Grenzen ihrer Vorgehensweise bewusst und plädiert in einem Ausblick selbst für eine umfassende sowie ländervergleichende Erhebung von Daten. Kropp, Buche und Buzogány geben eine aufschlussreiche und durch 90 leitfadengestützte Interviews methodisch fundierte Analyse der Transformation parlamentarisch-exekutiver Steuerungszusammenhänge sowie des Rollenverständnisses von Abgeordneten in Hinblick auf ihre Expertenrolle durch den Prozess der Europäisierung.

Daran schließt ein Abschnitt zu Zweiten Kammern und subnationalen Parlamenten an, womit der Blick auf einen der „blinden Flecken“ der bisherigen Forschung gerichtet wird. Zunächst gibt Anna Gamper einen informativen Überblick über die rechtliche Stellung Zweiter Kammern nach dem Vertrag von Lissabon: Sie kommt zu dem Schluss, dass sie leicht gestärkt werden, dies aber nicht zwangsläufig zu einer besseren Zusammenarbeit der verschiedenen Parlamente führt. Frank Delmartino erläutert das aus sieben unterschiedlichen föderalen und subnationalen Parlamenten bestehende belgische System, dessen Techniken der Zusammenarbeit in der Europaarbeit Aufmerksamkeit verdienen. Seine Verwendung des multi-level governance-Ansatzes zur Analyse der Beziehungen überrascht jedoch in einem Tagungsband, der mit dem Mehrebenenparlamentarismus explizit ein neues Konzept für die Zusammenarbeit von Parlamenten etablieren möchte. Abschließend untersucht Abels die Europaarbeit der deutschen Landtage in der Post-Lissabon-Phase. Mit Hilfe einer detaillierten Analyse der rechtlichen Entwicklung werden die großen bundesländerspezifischen Unterschiede in der Europapolitik sehr gut deutlich, die Frage der tatsächlichen Ausschöpfung dieser Rechte über das Fallbeispiel Baden-Württembergs hinaus bleibt künftiger Forschung vorbehalten.

Der letzte Abschnitt thematisiert die Möglichkeiten interparlamentarischer Vernetzung. Zunächst gibt Eppler einen Überblick über die Beziehungen, die zwischen den Parlamenten im EU-Mehrebenensystem bestehen können. Dabei benennt sie klar die methodologischen Herausforderungen, die in ihrem kurzen Beitrag nur bedingt bewältigt werden können. Henrik Scheller und Yvonne Eich befassen sich mit der virtuellen Vernetzung der Parlamente. Ihre Analyse zeigt, dass die vielfältigen Chancen für die Wahrnehmung einzelner parlamentarischer Funktionen, insbesondere im Hinblick auf direkte Dialogmöglichkeiten, von den 
einzelnen Parlamenten bisher nur unzureichend genutzt werden. Die von ihnen durch eine vermehrte virtuelle Präsenz erwartete Stärkung der demokratischen Legitimation der europäischen Politikgestaltung fällt, angesichts bekannter Repräsentativitätsprobleme und einer sich von anderen Institutionen und Akteuren durchaus unterscheidenden Parlamentsinstanz, jedoch allzu positiv aus.

Es bleibt festzuhalten, dass dieser informative Tagungsband umfangreich und vielseitig die Thematik des Parlamentarismus im EU-Mehrebenensystem erörtert und eine Vielzahl an neuen Erkenntnissen liefert. Die größte Herausforderung liegt nach wie vor in der umfassenden und systematischen empirischen Anwendung der vorliegenden theoretischen Konzepte, insbesondere des Mehrebenenparlamentarismus.

Somit bieten beide Bücher einen guten Überblick über den Stand der Parlamentarismusforschung in der EU. Während der Vorteil der Publikation von Dittgen in der kompakten Deskription der rechtlichen Situation nationaler Parlamente nach Inkrafttreten des Vertrags von Lissabon liegt, ist der Tagungsband von Abels und Eppler naturgemäß deutlich komplexer. Insbesondere die Beiträge der Praktiker (vor allem Preising und Vollrath) überzeugen durch ihr „Insider-Wissen“ zur politischen Praxis der verfassungsrechtlichen Neuerungen. Nun müssen weitere, insbesondere auch ländervergleichende Analysen erfolgen, die über deskriptive Fallstudien hinausgehen.

Ellen Schneider

\section{Antworten in der Rückschau, Fragen für die Zukunft der europäischen Integration}

Brincker, Gesa-Stefanie, Mathias Jopp und Lenka Anna Rovná (Hrsg.): Leitbilder for the Future of the European Union. Dissenting Promotors of Unity (Reihe Europäische Schriften, Band 90), Nomos Verlagsgesellschaft, Baden-Baden 2011, 420 Seiten, €69,-.

Stetter, Stephan, Carlo Masala und Marina Karbowski (Hrsg.): Was die EU im Innersten zusammenhält. Debatten zur Legitimität und Effektivität supranationalen Regierens (ZIB-Reader 01), Nomos Verlagsgesellschaft, Baden-Baden 2011, 374 Seiten, € 39,-.

Wie wird Europa in zwanzig oder dreißig Jahren aussehen? Diese „Gretchenfrage“ der europäischen Integration stellte Bundeskanzlerin Angela Merkel in ihrer Rede an der Prager Karls-Universität im April 2012. ${ }^{1}$ Die beiden vorliegenden Sammelbände nehmen sich der anspruchsvollen Aufgabe an, aus der Rückschau auf die politischen Entwicklungen der PostMaastricht-Ära ebenjene Frage nach den Perspektiven und Herausforderungen der europäischen Integration am Anfang des 21. Jahrhunderts näher zu beleuchten. Während der Fokus des Bandes von Gesa-Stefanie Brincker, Mathias Jopp und Lenka Anna Rovná dabei auf der Analyse des Einflusses europapolitischer Leitbilder ,as a key factor in the development of

1 Vgl. Angela Merkel, Rede bei der Diskussionsveranstaltung „Die künftige Gestalt Europas“ an der Juristischen Fakultät der Karls-Universität Prag am 3. April 2012, http://www.bundesregierung. de/Content/DE/Rede/2012/04/2012-04-03-merkel-prag.html (Abruf am 19. April 2012). 